\title{
Geranylgeranyl acetone prevents glutamate-induced cell death in HT-22 cells by increasing mitochondrial membrane potential
}

Eriko Sugano ${ }^{a^{*}}$, Yuka Endo ${ }^{a^{*}}$, Akihisa Sugai ${ }^{a}$, Yuki Kikuchia, Kitako Tabataa ${ }^{a}$, Taku Ozakia ${ }^{a}$ Takahiro Kurose $^{b}$, Yoshihiro Takai ${ }^{b}$, Yoko Mistsuguchi ${ }^{b}$, Yoichi Honma ${ }^{b}$, Hiroshi Tomita ${ }^{a, c, t}$

a Laboratory of Visual Neuroscience, Graduate Course in Biological Sciences, Iwate University Division of Science and Engineering, 4-3-5 Ueda, Morioka, Iwate 020-8551, Japan

b Rohto Pharmaceutical Co., Ltd, 6-5-4 Kunimidai, Kizugawa, Kyoto 619-0216, Japan

c Clinical Research, Innovation and Education Center, Tohoku University Hospital, 1-1 Seiryo, Aoba, Sendai, Miyagi 980-8574, Japan

*These authors contributed equally to this paper.

E-mail addresses: Eriko Sugano: sseriko@iwate-u.ac.jp, Yuka Endo: s0216006@iwate-u.ac.jp, Akihisa Sugai: nick341a@gmail.com, Yuki Kikuchi: s0217008@iwate-u.ac.jp, Kitako Tabata: ktabata@iwate-u.ac.jp, Taku Ozaki; tozaki@iwate-u.ac.jp, Takahiro Kurose: kurose@rohto.co.jp, Yoshihiro Takai: takai@rohto.co.jp, Yoko Mitsuguchi; mitsuguchi@rohto.co.jp, Yoichi Honma: honma@rohto.co.jp, Hiroshi Tomita: htomita@iwateu.ac.jp

${ }^{+}$Correspondence: Hiroshi Tomita, Ph.D., Iwate University Division of Science and Engineering, 43-5 Ueda, Morioka, Iwate 020-8551, Japan. Tel \& Fax: 81-19-621-6427; E-mail: htomita@iwate-u.ac.jp 


\begin{abstract}
Geranylgeranyl acetone (GGA) protects against various types of cell damages by upregulating heat shock proteins. We investigated whether GGA protect neuronal cells from cell death induced by oxidative stress. Glutamate exposure was lethal to HT-22 cells which comprise a neuronal line derived from mouse hippocampus. This configuration is often used as a model for hippocampus neurodegeneration in vitro. In the present study, GGA protected HT-22 cells from glutamate-induced oxidative stress. GGA pretreatment did not induce Hsps. Moreover, reactive oxygen species increased to the same extent in both GGA-pretreated and untreated cells exposed to glutamate. In contrast, glutamate exposure and GGA pretreatment increased mitochondrial membrane potential. However, increases in intracellular $\mathrm{Ca}^{2+}$ concentration were inhibited by GGA pretreatment. In addition, the increase of phosphorylated ERKs by the glutamate exposure was inhibited by GGA pretreatment. These findings suggest that GGA protects HT-22 cells from glutamate-provoked cell death without Hsp induction and that the mitochondrial calcium buffering capacity plays an important role in this protective effect.
\end{abstract}

Keywords: geranylgeranyl acetone (GGA), heat shock proteins (Hsps), HT-22 (hippocampal neuronal) cells, mitochondrial membrane potentials 


\section{Introduction}

Dementia is a brain disease that steadily reduces cognitive function and, by extension, quality of life. The number of patients with dementia is increasing rapidly in the aging society. However, no effective treatment has yet been developed for dementia. Glutamate is an excitatory neurotransmitter in the central nervous system and plays an important role in the development of dementia such as Alzheimer's disease [1]. Glutamate may also participates in the progression of cerebral ischemia and directly trigger neuronal cell death [2,3]. It was recently reported that glial cells release large amounts of glutamate which increases its overall concentration in the brain [4]. Other papers showed that the continuous activation of glutamate receptors is involved in excitation toxicities. Donepezil is a cholinesterase inhibitor that suppresses decreases in acetylcholine neurotransmitter concentration. It was developed to treat dementia based on earlier reports that brain acetylcholine levels decline in Alzheimer's disease [5]. The $N$-methyl- $D$-aspartate (NMDA) receptor antagonist memantine was formulated in response to the hypothesis that neuronal cell death is caused by excessive brain glutamate [6]. Both of these agents may delay the progression of these diseases but cannot cure them. Glutamate may act on AMPA- and kainite- as well as NMDA receptors. Excessive receptor activation may result in cell death $[7,8]$. Therefore, new drugs with novel modes of action are required for the effective treatment of dementia.

Another putative cell death pathway is glutamate-mediated inhibition of glutathione (GSH) synthesis [9]. A cystine-glutamate transporter in neurons is responsible for the uptake of cystine from the extracellular space and its exchange for intracellular glutamate. Once incorporated, the cystine is reduced to cysteine and used to synthesize GSH for oxidative stress reduction [10]. HT-22 are neuronal cells derived from mouse hippocampus. They are susceptible to glutamate toxicity. HT-22 cells lack NMDA-type glutamate receptors. Therefore, glutamate toxicity occurs in HT-22 cells via cystineglutamate transporter inhibition [11].

Ischemic preconditioning suppresses neuronal cell death by inducing ischemic tolerance via mild preliminary ischemic invasion [12]. Ischemic preconditioning is protective because it upregulates heat shock proteins (Hsps) [13,14]. Geranylgeranyl acetone (GGA) is an acyclic isoprenoid compound used to treat gastritis and gastric ulcer. It was also the first drug confirmed to induce Hsps in the gastric mucosa [15]. Hsps are synthesized under thermal, physiological, and biological stress conditions. They fold newly synthesized proteins, transport them across membranes, and refold misfolded or aggregated proteins [16]. GGA may be cytoprotective by inducing Hsp70 overexpression which, in turn, suppresses apoptosis by inhibiting the release of cytochrome $c$ and apoptosis-inducing factors and suppressing JNK phosphorylation. In this way, procaspase 9 is activated [17-19].

We hypothesized that GGA protects against neuronal cell death by inducing Hsps. In the present study, however, we showed that GGA did not, in fact, induce HSPs to protect HT-22 cells against glutamate-induced toxicity. Subsequent investigations of ROS production, changes in membrane potentials and intracellular $\mathrm{Ca}^{2+}$ concentration, and mitochondrial protein expression revealed that a major component of the protective effect of GGA consisted of increasing mitochondrial membrane potential. 


\section{Results}

\section{Protective effect of GGA against glutamate-induced cell death}

HT-22 cell viability decreased in a dose-dependent manner in response to the addition of glutamate to the culture medium. The TD 50 was $\sim 1.5 \mathrm{mM}$ (Fig. 1A). Survival rates continued to rise significantly up to $200 \mu$ M GGA. At this concentration, cell viability was $\sim 80 \%$ (Fig. 1B). The number of HT-22 cells decreased $24 \mathrm{~h}$ after glutamate exposure. They presented with characteristic atrophy (Fig. 1C). On the other hand, $300 \mu \mathrm{M}$ GGA had no protective effect. Higher GGA concentrations may have actually been somewhat toxic to the HT-22 cells. To confirm this toxicity, HT-22 cells were cultured with various GGA concentrations in the absence of glutamate. Cell viability significantly decreased at GGA concentrations $>500 \mu \mathrm{M}$ (Fig. 1D and 1E).

\section{Hsps expressions induced by GGA}

To establish whether the protective effect of GGA on glutamate-exposed HT22 cells was related to Hsp induction, Hsp70 and Hsp90 levels were analyzed by western blot (Fig. 2A) and RT-PCR (Fig. 2B). The protein levels of Hsp70 and Hsp90 did not significantly change in response to GGA treatment (Fig. 2A and 2B). Moreover, neither did their gene expression levels.

\section{ROS measurement}

We measured ROS production in HT-22 cells treated with glutamate. ROS levels significantly increased in both GGA- and glutamate-treated HT-22 cells. The ROS levels in GGA-treated HT-22 cells were the same as those in glutamate-treated HT-22 cells. GGA and glutamate treatments also enhanced de novo ROS production (Fig. 3A and 3B). Thus, GGA did not suppress ROS induced by glutamate toxicity.

\section{Mitochondrial membrane potential measurement}

Mitochondrial membrane potential was measured every $6 \mathrm{~h}$ after adding glutamate. The mitochondrial membrane potentials gradually increased in the HT-22 cells treated with glutamate and reached a maximum at $12 \mathrm{~h}$ after treatment. On the other hand, mitochondrial membrane potentials immediately increased in response to GGA whether or not glutamate was present. Moreover, their levels remained high throughout the experimental period (Fig. 4A and 4B).

\section{Changes in $\mathrm{Ca}^{2+}$ concentration ERK phosphorylation}

Changes in intracellular $\mathrm{Ca}^{2+}$ concentration were measured $8 \mathrm{~h}$ after glutamate exposure. Calcium concentration significantly increased in HT-22 cells exposed to glutamate. However, this increase was prevented by GGA pretreatment (Fig. 5A and 5B). The phosphorylated levels of ERKs increased by the glutamate exposure that inhibited by GGA pretreatment though the ERKs levels were not changed (Fig. $6 \mathrm{~A}$ and $6 \mathrm{~B})$. 
A

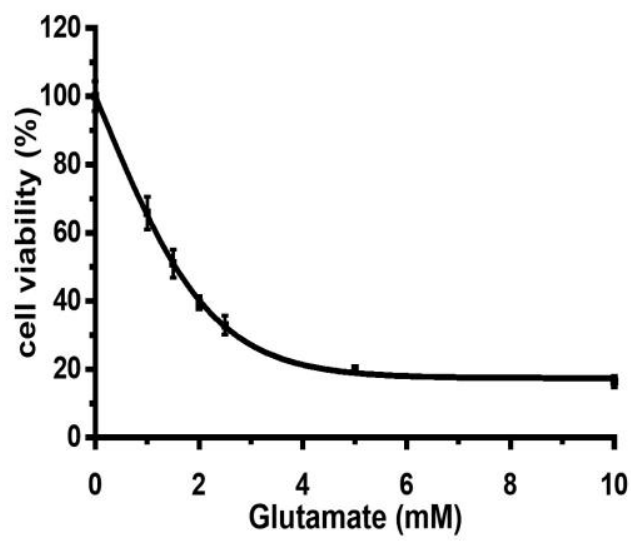

B

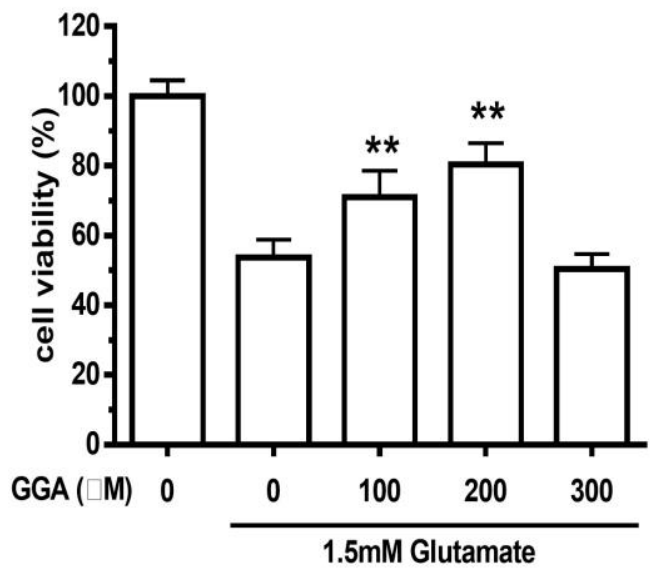

C

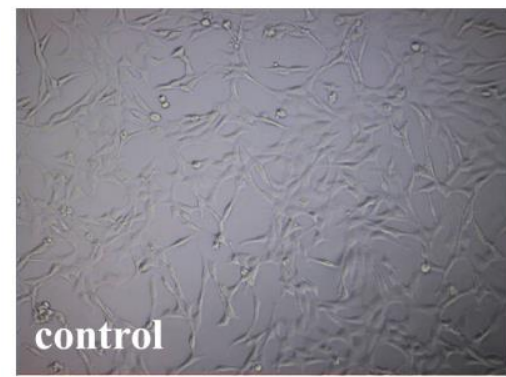

D

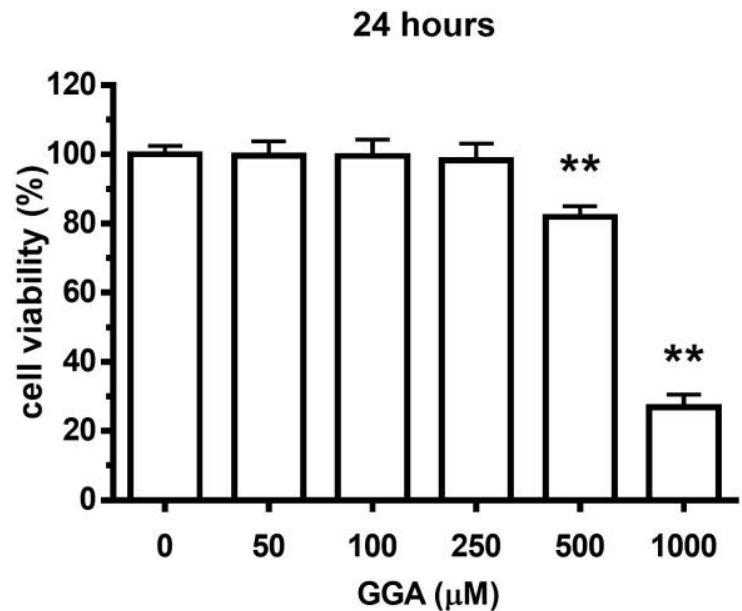

E

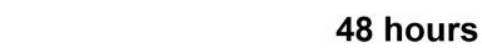

Fig. 1. Protective effect of GGA against glutamate toxicity in HT-22 cells. Glutamate-induced cytotoxicity in HT-22 cells was evaluated $24 \mathrm{~h}$ after glutamate addition (A). HT-22 cells were preincubated with GGA for $24 \mathrm{~h}$ and exposed to $1.5 \mathrm{mM}$ glutamate for $24 \mathrm{~h}$ (B). Cell viabilities increased at after pre-incubation with $100 \mu \mathrm{M}$ or $200 \mu \mathrm{M}$ GGA. Cells became atrophic in response to glutamate exposure $(C)$. Higher GGA concentrations were cytotoxic $(D, E)$. Data are means $\pm S D$. $(n=5$; Dunnett's multiple comparison test; $\left.{ }^{* *} P<0.01\right)$. 
A

Hsp 70

Hsp 90

$\beta$-actin
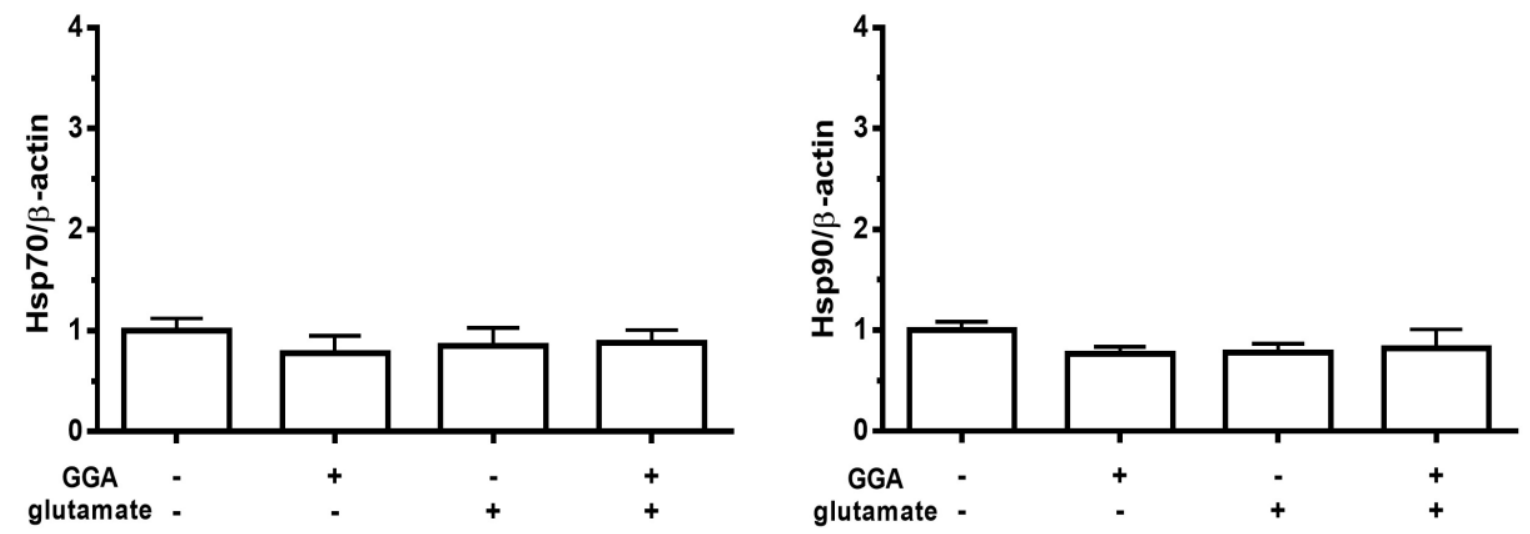

B
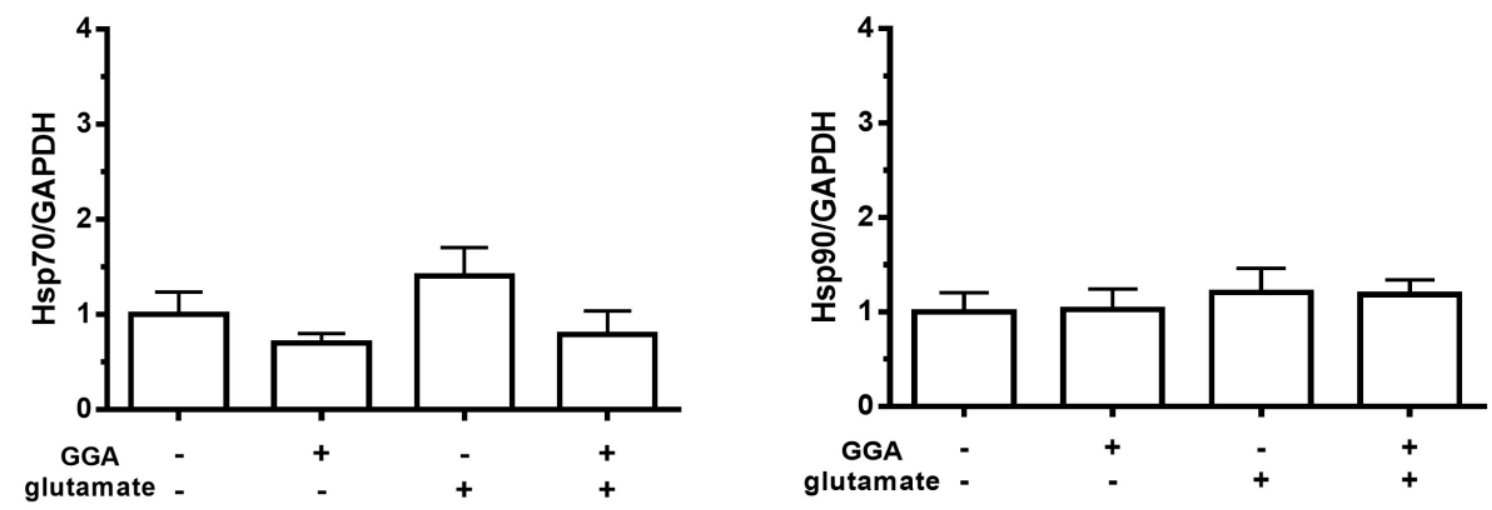

Fig. 2. Hsp70 and Hsp90 expression in HT-22 cells. Hsp70 and Hsp90 protein expression levels were quantified by western blot. They were extracted immediately from untreated HT-22 cells and from the other HT-22 cells $6 \mathrm{~h}$ after glutamate exposure (A, B). Band densities were normalized with those of $\beta$ actin on the same blots. There were no significant difference among groups $(n=4)$. The mRNA expression levels of Hsp70 and Hsp90 were measured by RT-PCR (C, D). Data were normalized with GAPDH expression $(n=3)$. The GGA and glutamate concentrations were $200 \mu \mathrm{M}$ and $1.5 \mathrm{mM}$, respectively. 


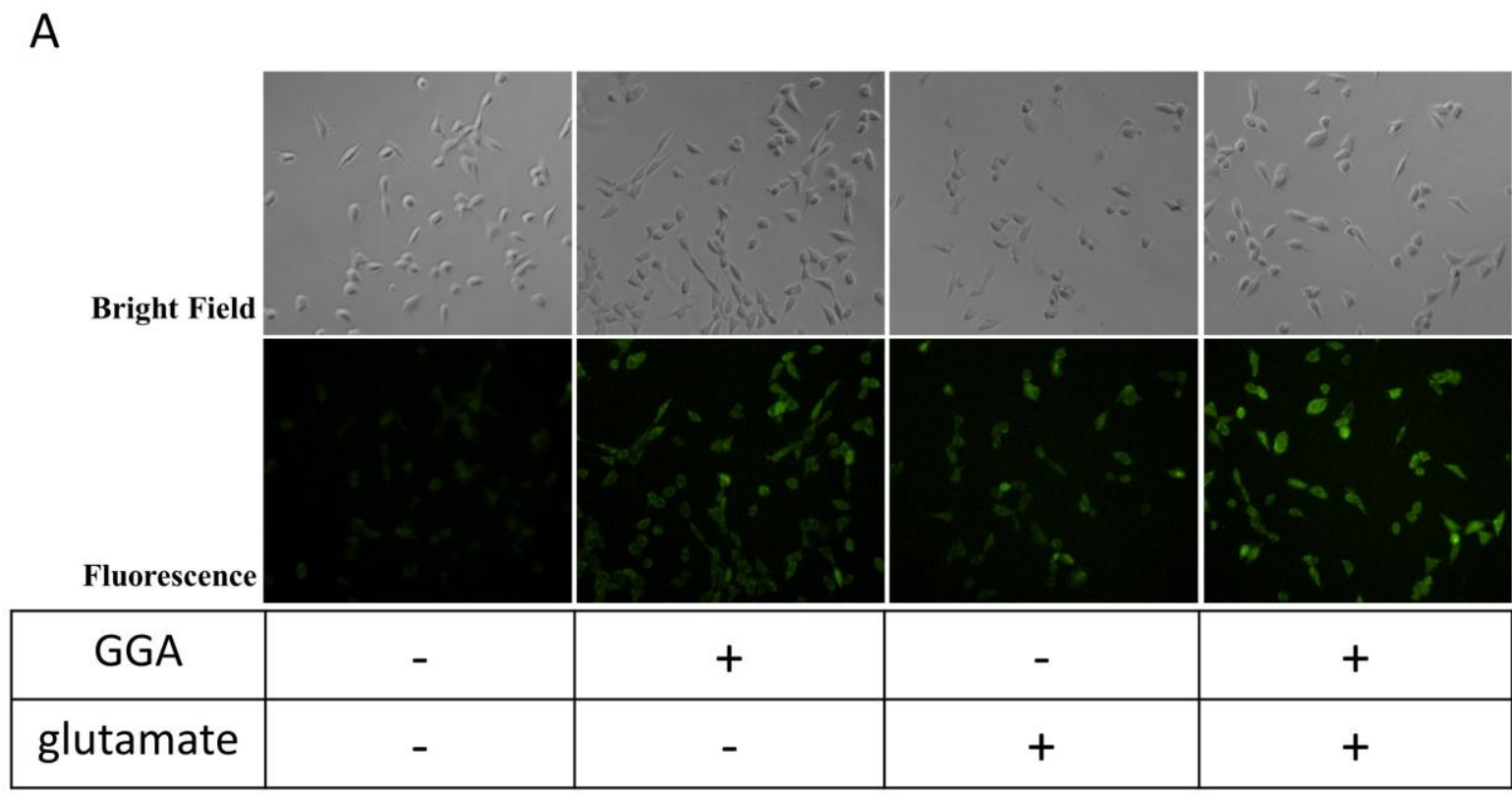

B

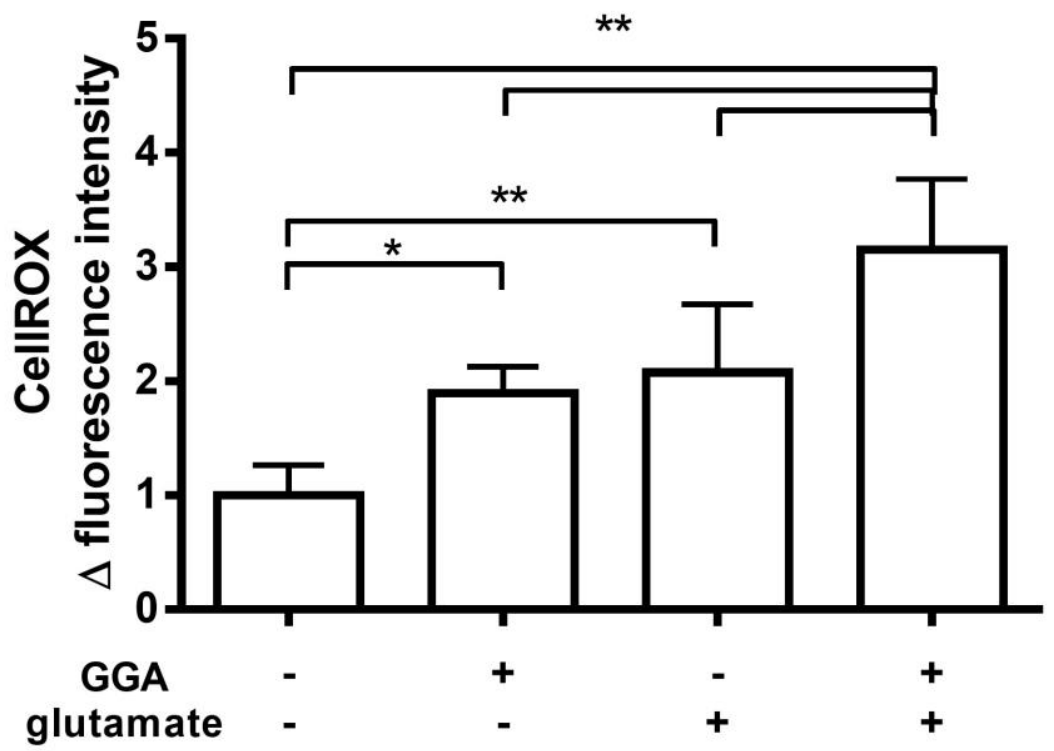

Fig. 3. ROS levels in HT-22 cells $6 \mathrm{~h}$ after glutamate exposure. HT-22 cells exposed to glutamate were still alive $6 \mathrm{~h}$ later, as shown in the bright field photograph. Fluorescence images clearly showed increased ROS in both untreated HT-22 cells and in those treated with GGA and/or glutamate (A). ROS levels increased in all treated groups relative to those in untreated HT-22 cells (B). Data are means \pm SD ( $\mathrm{n}=6$; Dunnett's multiple comparison test; ${ }^{*} P<0.05 ;{ }^{* *} P<0.01$ ). 
A

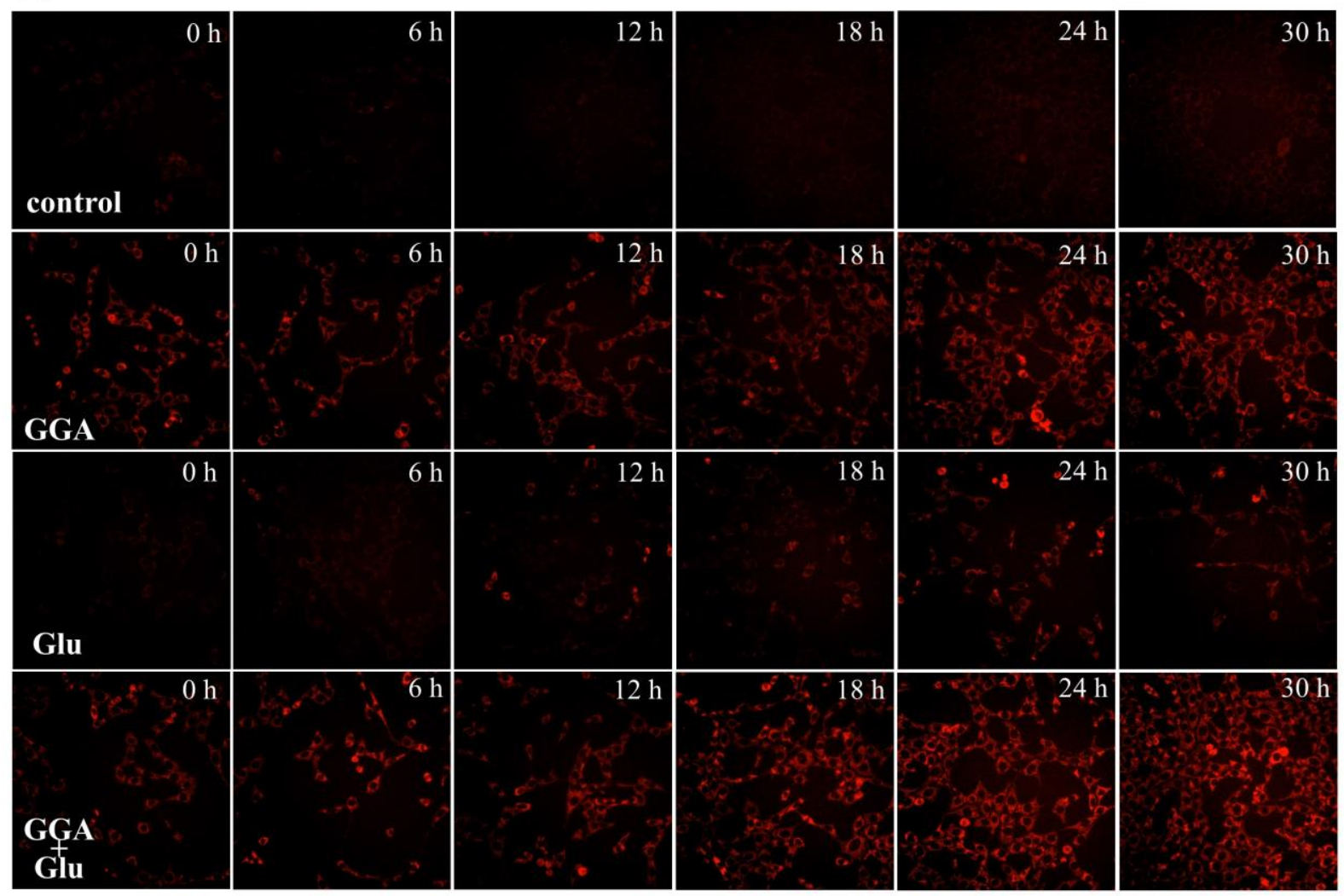

B

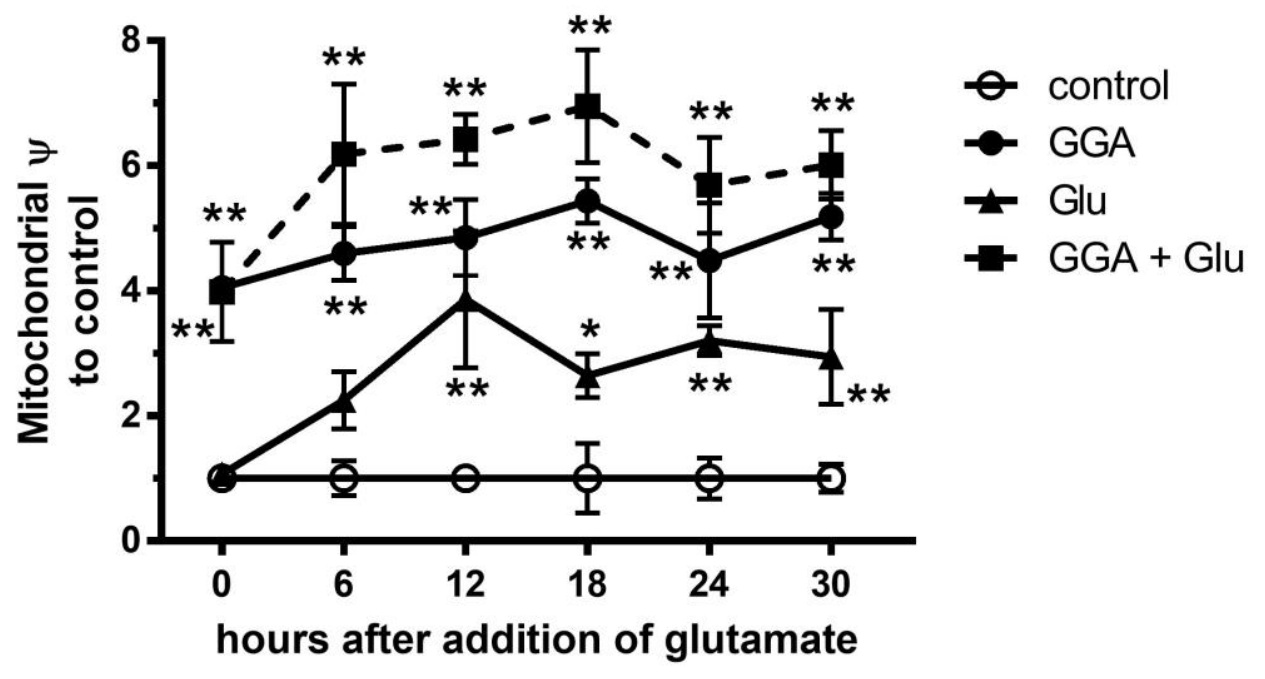

Fig. 4. Changes in HT-22 mitochondrial membrane potential after glutamate exposure. TMRM fluorescence images taken at $0 \mathrm{~h}, 6 \mathrm{~h}, 12 \mathrm{~h}, 18 \mathrm{~h}, 24 \mathrm{~h}$, and $30 \mathrm{~h}$ after glutamate addition (A). Mitochondrial membrane potentials of cells pretreated with GGA had already increased by the time the glutamate was added (B). Fluorescence intensities of the treated HT-22 cells were compared to those of the control at each time point. ( $\mathrm{n}=3$; Tukey's test, ${ }^{* *} P<0.01$; mean $\pm \mathrm{SD}$ ). 
A

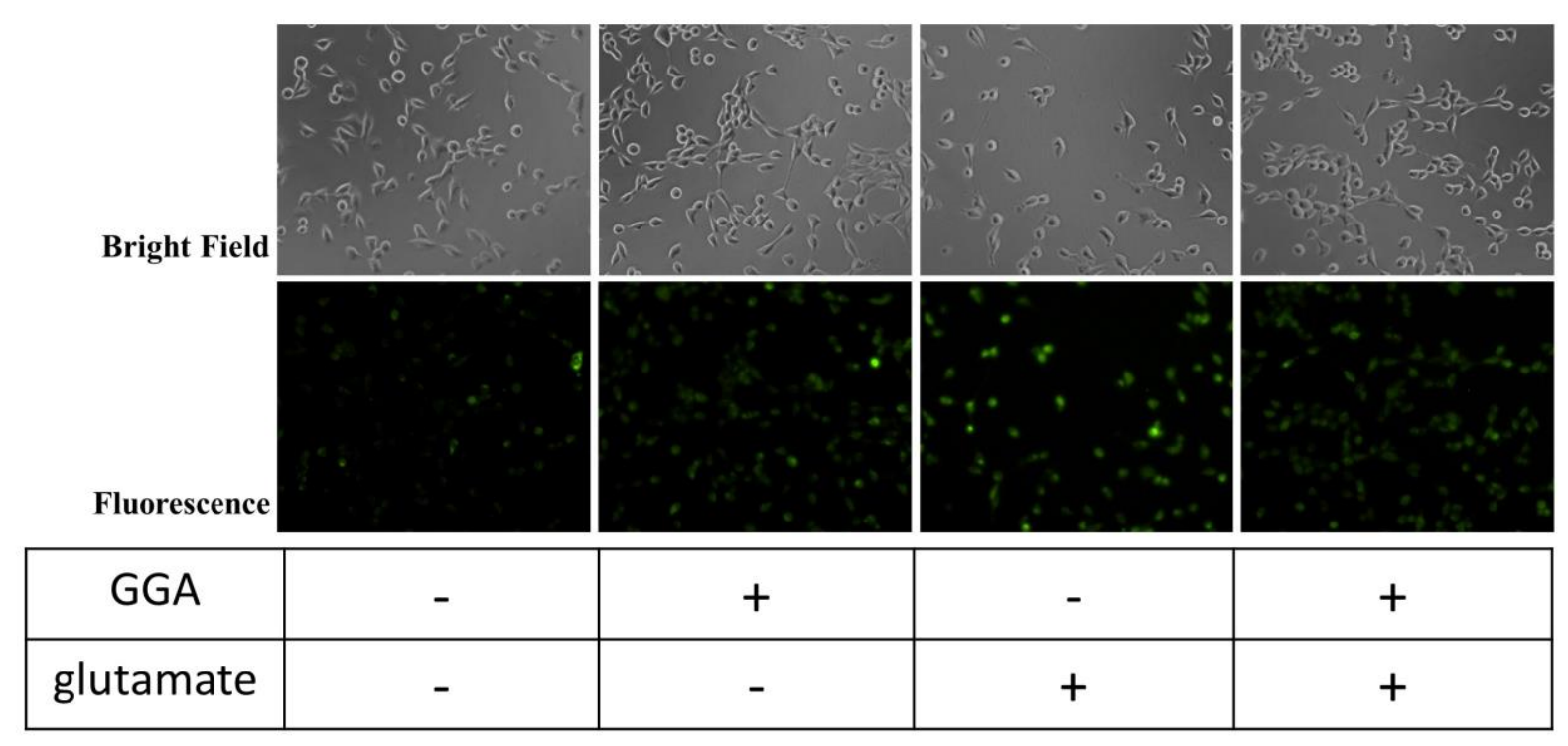

B

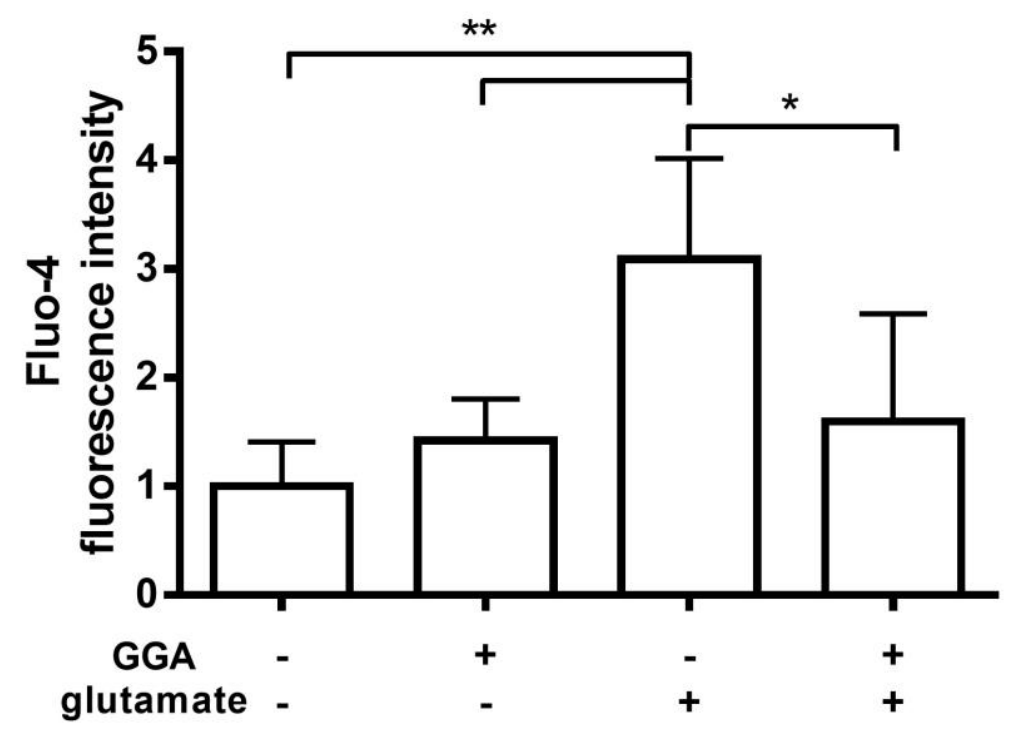

Fig. 5. $\mathrm{Ca}^{2+}$ concentrations in HT-22 cells $8 \mathrm{~h}$ after glutamate exposure. Fluo-4 fluorescence images were taken $8 \mathrm{~h}$ after glutamate addition. Bright field- and fluorescent photographs of each group are shown in A. Fluorescence intensities in each group were quantified. Data are means $\pm S D ; n=6$; Dunnett's multiple comparison test; ${ }^{*} P<0.05 ;{ }^{* *} P<0.01$ ). 
A
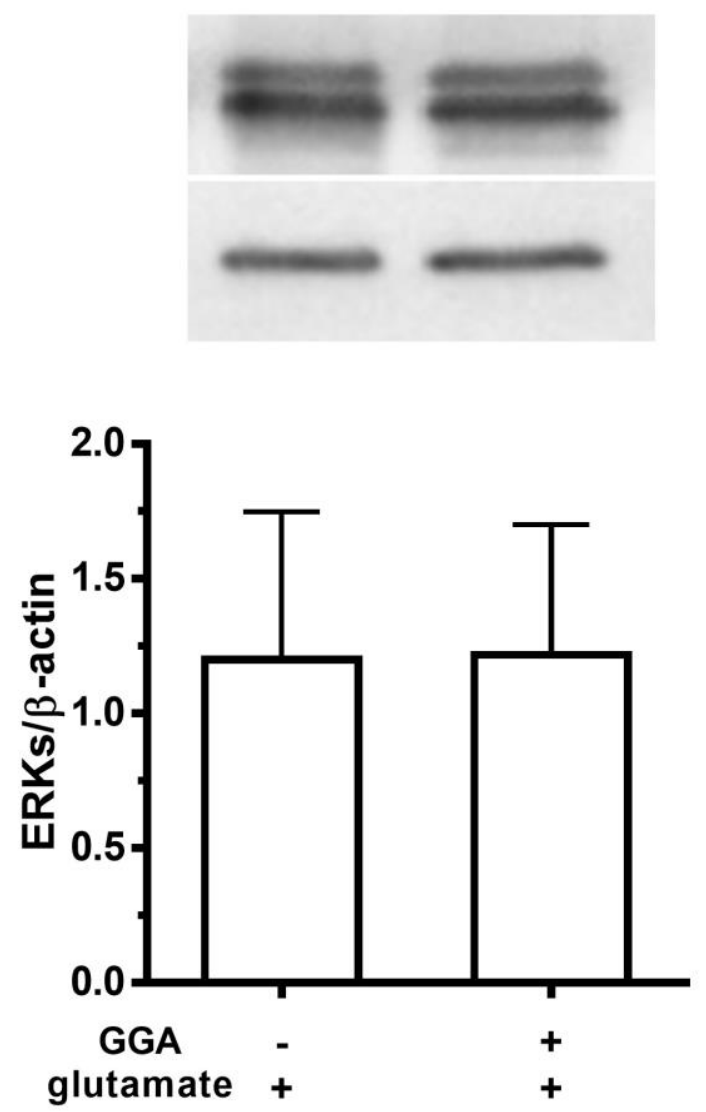

B

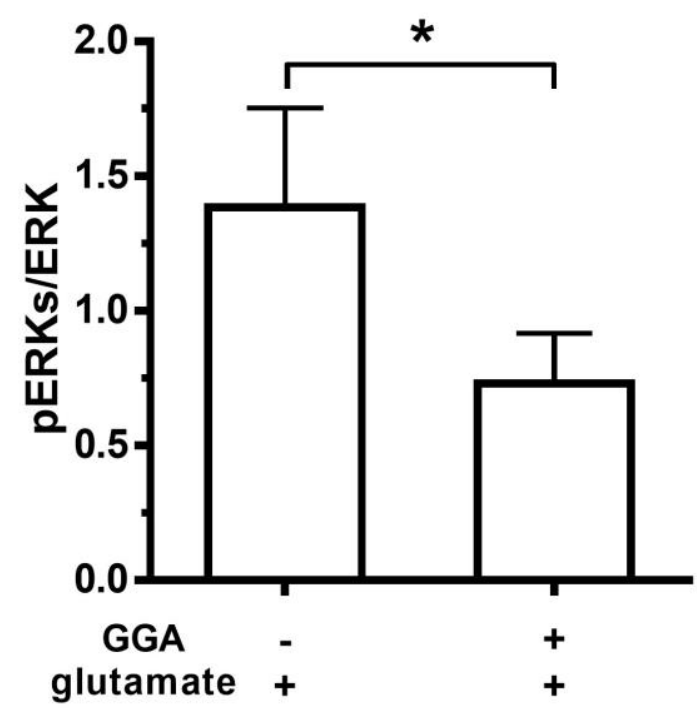

Fig. 6. Western blot of phosphorylated ERK1/2 in HT-22 cells. There was no difference in ERKs level between glutamate-exposed HT-22 cells with or without GGA. Phosphorylated ERKs in glutamateexposed HT-22 cells significantly decreased in response to GGA treatment $\left(\mathrm{n}=4\right.$; Student's t-test, ${ }^{*} \mathrm{P}<$ 0.05 ; mean $\pm \mathrm{SD}$ ).

\begin{tabular}{|c|c|c|}
\hline Name & \multicolumn{1}{|c|}{ Sequence $\left(5^{\prime} \rightarrow \mathbf{3}^{\prime}\right)$} & Genbank \\
\hline \multirow{2}{*}{ mouse-GAPDH } & F : AGGTCGGTGTGAACGGATTTG & \multirow{2}{*}{ NM_008084 } \\
\cline { 2 - 2 } & R : TGTAGACCATGTAGTTGAGGTCA & \\
\hline \multirow{2}{*}{ mouse-Hsp70a } & F : TGGTGCAGTCCGACATGAAG & \multirow{2}{*}{ NM_010479 } \\
\cline { 2 - 3 } & R : GCTGAGAGTCGTTGAAGTAGGC & NM_010480 \\
\hline mouse-Hsp90 $\alpha$ & F : TGTTGCGGTACTACACATCTGC &
\end{tabular}

Table 1 List of Primer pairs

\begin{tabular}{|c|c|c|}
\hline Antigen & Source & Manufactuer \\
\hline Hsp70 & mouse & Abcam (ab2787) \\
\hline Hsp90 & rabbit & Cell Signaling (\#4877) \\
\hline ERK & rabbit & Cell Signaling(\#4695) \\
\hline
\end{tabular}




\begin{tabular}{|c|c|c|} 
pERK & rabbit & Cell Signaling(\#9101) \\
\hline$\beta$-actin & mouse & Santa Cruz biochnology (sc-69879) \\
\hline Anti-Rabbit IgG & rabbit & Promega (S3731) \\
\hline Anti-Mouse IgG & mouse & Promega (S3721) \\
\hline
\end{tabular}

Table 2 List of Antibodies

\section{Discussion}

Glutamate-induced cytotoxicity in HT-22 cells is associated with oxidative stress. This model is often used to evaluate the neuroprotective efficacies of various pharmaceutical agents. Here, we demonstrated the protective effects of GGA against the death of HT-22 hippocampal neuronal cells (Fig. 1B). GGA is clinically administered to treat gastritis and gastric ulcer. It induces Hsp expression and protects gastric mucosal cells [20]. The protective effects of GGA-mediated Hsp induction were also reported for myocardial [21], hepatic [22], and retinal [23] cells. In the present study, no Hsp induction was observed in the HT-22 cells (Fig. 2A, B). Cisplatin-induced reduction in cell viability was attenuated by suppressing intracellular p53 without GGA-mediated Hsp induction [24]. Therefore, the mechanism by which GGA protects against glutamate-induced HT-22 cell death may involve pathways independent of Hsp induction.

Morphological changes in HT-22 cells were observed $6 \mathrm{~h}$ after glutamate exposure (Supplementary Video 1). The critical time to assess HT-22 cell death was $\sim 6 \mathrm{~h}$ after glutamate addition. Increases in ROS directly induce cell death. Antioxidants protect cells from oxidative stress [25-27]. Thus, ROS was also measured at $6 \mathrm{~h}$ after glutamate treatment. Marked increases in ROS were observed in HT-22 cells treated with GGA and glutamate (Fig. 3). The cytoprotective effect of GGA is not associated with ROS level as both GGA and glutamate exposures enhanced ROS production.

To elucidate the protective mechanisms of GGA, we measured the mitochondrial membrane potentials in HT-22 cells. Mitochondria play important roles in neuronal cell death [28]. GGA steadily increased HT-22 mitochondrial membrane potentials during the observation periods (Fig. 4B). Increases in mitochondrial membrane potential may serve to buffer calcium in mitochondria and delay hippocampal neuron degeneration [29]. The calcium uptake activity of the MCU (mitochondrial calcium uniporter) localized in the inner mitochondrial membrane varies with $\mathrm{Ca}^{2+}$ concentration outside the mitochondria. MCU regulates intracellular calcium concentrations [30,31]. It is thought that the mitochondrial membrane potential drives calcium uptake by this organelle according to the Nernst equation [32]. Therefore, calcium buffering enhancement effected by raising the mitochondrial membrane potential may play a crucial role in the cytoprotective effect of GGA. We then investigated the intracellular $\mathrm{Ca}^{2+}$ concentration. A significant increase in intracellular $\mathrm{Ca}^{2+}$ was observed after $8 \mathrm{~h}$ following glutamate exposure as previously reported [33]. The increase in intracellular $\mathrm{Ca}^{2+}$ was suppressed by GGA addition. Intracellular $\mathrm{Ca}^{2+}$ concentration was affected by ERKs activation associated with glutamate-induced HT-22 cell death [11,34,35]. In the present study, GGA administration to glutamate-exposed HT-22 cells inhibited ERKs phosphorylation (Figure 6). Thus, increases in the membrane potential of the mitochondria enhanced their calcium buffering capacity and suppressed the cell death pathway associated with elevated $\mathrm{Ca}^{2+}$ concentration in HT-22 cells.

In summary, glutamate exposure in HT-22 cells caused oxidative stress as manifested by relative increases in ROS production. GGA induced ROS generation but did not scavenge free radicals or induce Hsp. In the early phase of glutamate toxicity, however, GGA treatment increased mitochondrial membrane potential. In this manner, the mitochondrial calcium buffering capacity was enhanced as a defense against ROS-induced calcium overload. Therefore, GGA could be repurposed as a dementia therapy. Moreover, this study disclosed that $\mathrm{Ca}^{2+}$ overload is implicated in the cell death pathways associated with various excitotoxicity disorders such as ischemia/reperfusion injury. 


\section{Materials and Methods}

Reagents

GGA (Tokyo Chemical Industry Co. Ltd., Tokyo, Japan) was dissolved in dimethyl sulfoxide (DMSO; Sigma-Aldrich Corp., Tokyo, Japan) to prepare a $1 \mathrm{M}$ solution. This stock would then be diluted to the desired concentrations with Dulbecco's modified Eagle's medium. L-glutamic acid-sodium (Nacalai Tesque, Kyoto, Japan) was dissolved in the culture medium and diluted to the desired concentrations.

\section{Cell culture}

HT-22 cells (a murine hippocampal-derived neuronal cell line) were cultured with Dulbecco's modified Eagle's medium (DMEM; Thermo Fisher Scientific, Tokyo, Japan) supplemented with 10\% (v/v) fetal bovine serum (FBS), 1\% (w/v) antibiotics (Thermo Fisher Scientific, Tokyo, Japan), 1\% (w/v) Glutamax (Thermo Fisher Scientific, Tokyo, Japan) and 0.35\% (w/v) D-glucose (Wako Pure Chemical Industries Ltd., Tokyo, Japan) at $37{ }^{\circ} \mathrm{C}$ under a $5 \% \mathrm{CO}_{2}$ atmosphere. The cells were maintained by exchanging the culture medium every $2 \mathrm{~d}$ and passaging with $0.25 \%(\mathrm{w} / \mathrm{v})$ trypsin/ $1 \mathrm{mM}$ EDTA-2Na in Dulbecco's phosphate-buffered saline (DPBS). HT-22 cells were seeded at a density of $5 \times 10^{3}$ well ${ }^{-1}$ in a 96-well plate $1 \mathrm{~d}$ before glutamate addition. Various glutamate concentrations were added to the media, the cells were incubated for $24 \mathrm{~h}$, and their viabilities were evaluated with an aqueous cell titer reagent (Promega, Tokyo, Japan) followed the manufacturer's protocol. In a separate assay, GGA was added to the culture medium $24 \mathrm{~h}$ before glutamate exposure.

\section{RT-PCR}

Total RNA was extracted with an Absolutely RNA Microprep Kit (Agilent Technologies, Tokyo, Japan) at 24 hours after the glutamate exposure from untreated cells and from those exposed to glutamate and/or GGA. The cDNA was synthesized using a ReverTra Ace ${ }^{\circledR}$ qRT-PCR Master Mix with gDNA Remover (TOYOBO, Tokyo, Japan). Hsp70, Hsp90, and GAPDH expression levels were quantified by a CFX Connect ${ }^{\mathrm{TM}}$ Real-Time PCR Analysis System (Bio-Rad Laboratories, Tokyo, Japan) using the primer pairs listed in Table 1. RT-PCR was performed using SsoAdvanced ${ }^{\mathrm{TM}}$ Universal SYBR $^{\circledR}$ Green Supermix (Bio-Rad Laboratories, Tokyo, Japan) according to the manufacturer's instructions. GAPDH was used as a reference gene and quantification was conducted by the comparative Ct method.

\section{Western blot}

HT-22 cells were seeded into 6-cm plates at a density of $3.5 \times 10^{5}$ cells per plate with or without GGA. After $24 \mathrm{~h}$, glutamate was added to the cells. After $6 \mathrm{~h}$, the proteins were extracted with radioimmunoprecipitation assay (RIPA) buffer (Thermo Fisher Scientific, Tokyo, Japan) including Halt $^{\mathrm{TM}}$ protease and EDTA-free phosphatase inhibitor single-use cocktail (Thermo Fisher Scientific, Tokyo, Japan). Protein concentrations were measured with a bicinchoninic acid (BCA) protein assay kit (Thermo Fisher Scientific, Tokyo, Japan). Twenty micrograms of protein was loaded onto each lane on a 4-15\% miniprotean TGX precast polyacrylamide gel (Bio-Rad Laboratories, Tokyo, Japan) and transferred to a polyvinylidene fluoride (PVDF) membrane. After blocking, the membrane was incubated with primary antibody. After washing, the membrane was incubated with secondary alkaline phosphatase-conjugated antibody. Chemiluminescence detection (CDP-Star detection reagent; GE Healthcare, Tokyo, Japan) was performed according to the standard procedure. Band densities were measured in ImageQuant (GE Healthcare, Tokyo, Japan). The density of each band was normalized to that of $\beta$-actin. The antibodies used in this study are listed in Table 2.

Measurements of ROS, mitochondrial membrane potentials, and $\mathrm{Ca}^{2+}$ concentrations

Changes in ROS, mitochondrial membrane potential, and $\mathrm{Ca}^{2+}$ concentration were detected with CellROX Green Reagent (Thermo Fisher Scientific, Tokyo, Japan), tetramethylrhodamine methyl ester 
(TMRM; Thermo Fisher Scientific, Tokyo, Japan), and Fluo-4 (Thermo Fisher Scientific, Tokyo, Japan), respectively, following the manufacturer's instructions. Fluorescence images were viewed and photographed under a fluorescence microscope (Apotome-2; Carl Zeiss, Tokyo, Japan). Fluorescence intensities were measured in ImageJ ver.1.80 (NIH, Bethesda, MD, USA).

Statistical analyses

Data were processed in GraphPad Prism ver. 6.02 (MDF, Tokyo, Japan). Treatment means were compared by Tukey's and Dunnett's multiple comparison tests and Student's t-test. 


\section{Supplementary Materials}

Vid. S1. Time lapse of growing HTT-22 cells in medium containing glutamate with or without GGA. Time lapse images were taken with JuLI FL (AR BROWN Co. Ltd, Tokyo, Japan). Left and right panels show HT-22 cells cultured in medium containing $1.5 \mathrm{mM}$ glutamate and $200 \mu \mathrm{M}$ GGA or no GGA, respectively.

\section{Funding}

This work was supported by Grants-in-Aid for Scientific Research from the Ministry of Education, Culture, Sports, Science and Technology, Japan (Nos. 19H03807, 19K09945, 18K09433, and 17H06330).

\section{Acknowledgments}

The authors thank Mses. Yoko Takahashi, Misao Enomoto, Miho Sato, and Takako Kishimoto of the Laboratory of Visual Neuroscience for maintaining the cultures of the HT-22 cells used in this study.

\section{Author Contributions}

H.T. conceptualized the study. H.T., T. K., Y.T., Y. M., and Y.H developed the methodology. K.T. and T.O validated the methodology. A.S. formally analyzed the data. Y.E., E.S. and A.S. curated the data. A.S. prepared the original manuscript draft. H.T and E.S. reviewed and edited the manuscript. H.T. was responsible for visualization. H.T., E.S., and K.T. procured funding for the study.

\section{Conflicts of Interest}

Messrs. Takahiro Kurose, Yoshihiro Takai, and Yoichi Honma are employed by Rohto Pharmaceutical Co. Ltd., Osaka, Japan. The other authors declare that they have no competing interests.

\section{Abbreviations \\ GGA Geranylgeranyl acetone \\ HSP Heat shock protein \\ HT-22 hippocampal neuronal cells}

\section{Reactive Oxygen Species ROS}

\section{References}

1. Coyle, J.T.; Puttfarcken, P. Oxidative stress, glutamate, and neurodegenerative disorders. Science 1993, 262, 689-695.

2. Dong, X.X.; Wang, Y.; Qin, Z.H. Molecular mechanisms of excitotoxicity and their relevance to pathogenesis of neurodegenerative diseases. Acta Pharmacol Sin 2009, 30, 379-387, doi:aps200924 [pii]. 10.1038/aps.2009.24.

3. Nicholls, D.; Attwell, D. The release and uptake of excitatory amino acids. Trends Pharmacol Sci 1990, 11, 462468, doi:0165-6147(90)90129-V [pii].

4. Beppu, K.; Sasaki, T.; Tanaka, K.F.; Yamanaka, A.; Fukazawa, Y.; Shigemoto, R.; Matsui, K. Optogenetic countering of glial acidosis suppresses glial glutamate release and ischemic brain damage. Neuron 2014, 81, 314-320, doi:S0896-6273(13)01079-9 [pii]. 10.1016/j.neuron.2013.11.011.

5. Lee, J.H.; Jeong, S.K.; Kim, B.C.; Park, K.W.; Dash, A. Donepezil across the spectrum of Alzheimer's disease: dose optimization and clinical relevance. Acta Neurol Scand 2015, 131, 259-267, doi:10.1111/ane.12386.

6. van Wageningen, H.; Jorgensen, H.A.; Specht, K.; Eichele, T.; Hugdahl, K. The effects of the glutamate antagonist memantine on brain activation to an auditory perception task. Hum Brain Mapp 2009, 30, 36163624, doi:10.1002/hbm.20789. 
7. Furukawa, T.; Hoshino, S.; Kobayashi, S.; Asakura, T.; Takahashi, M.; Atsumi, T.; Teramoto, A. The glutamate AMPA receptor antagonist, YM872, attenuates cortical tissue loss, regional cerebral edema, and neurological motor deficits after experimental brain injury in rats. J Neurotrauma 2003, 20, 269-278, doi:10.1089/089771503321532851.

8. Westergren, I.; Johansson, B.B. NBQX, an AMPA antagonist, reduces glutamate-mediated brain edema. Brain Res 1992, 573, 324-326, doi:0006-8993(92)90781-4 [pii].

9. Tan, S.; Schubert, D.; Maher, P. Oxytosis: A novel form of programmed cell death. Curr Top Med Chem 2001, 1, 497-506.

10. Murphy, T.H.; Miyamoto, M.; Sastre, A.; Schnaar, R.L.; Coyle, J.T. Glutamate toxicity in a neuronal cell line involves inhibition of cystine transport leading to oxidative stress. Neuron 1989, 2, 1547-1558, doi:08966273(89)90043-3 [pii].

11. Fukui, M.; Song, J.H.; Choi, J. Choi, H.J.; Zhu, B.T. Mechanism of glutamate-induced neurotoxicity in HT22 mouse hippocampal cells. Eur J Pharmacol 2009, 617, 1-11, doi:S0014-2999(09)00578-0 [pii]. 10.1016/j.ejphar.2009.06.059.

12. Nishiyama, T.; Nishukawa, S.; Hiroshi; Tomita; Tamai, M. Muller cells in the preconditioned retinal ischemic injury rat. Tohoku J Exp Med 2000, 191, 221-232.

13. Doeppner, T.R.; Doehring, M.; Kaltwasser, B.; Majid, A.; Lin, F.; Bahr, M.; Kilic, E.; Hermann, D.M. Ischemic post-conditioning induces post-stroke neuroprotection via Hsp70-mediated proteasome inhibition and facilitates neural progenitor cell transplantation. Mol Neurobiol 2017, 54, 6061-6073, doi:10.1007/s12035-0160137-3. 10.1007/s12035-016-0137-3 [pii].

14. Liu, Y.; Kato, H.; Nakata, N.; Kogure, K. Temporal profile of heat shock protein 70 synthesis in ischemic tolerance induced by preconditioning ischemia in rat hippocampus. Neuroscience 1993, 56, 921-927, doi:03064522(93)90138-6 [pii].

15. Hirakawa, T.; Rokutan, K.; Nikawa, T.; Kishi, K. Geranylgeranylacetone induces heat shock proteins in cultured guinea pig gastric mucosal cells and rat gastric mucosa. Gastroenterology 1996, 111, 345-357, doi:S0016508596003538 [pii].

16. Bukau, B.; Weissman, J.; Horwich, A. Molecular chaperones and protein quality control. Cell 2006, 125, 443451, doi:S0092-8674(06)00495-8 [pii]. 10.1016/j.cell.2006.04.014.

17. Beere, H.M.; Wolf, B.B.; Cain, K.; Mosser, D.D.; Mahboubi, A.; Kuwana, T.; Tailor, P.; Morimoto, R.I.; Cohen, G.M.; Green, D.R. Heat-shock protein 70 inhibits apoptosis by preventing recruitment of procaspase- 9 to the Apaf-1 apoptosome. Nat Cell Biol 2000, 2, 469-475, doi:10.1038/35019501.

18. Ravagnan, L.; Gurbuxani, S.; Susin, S.A.; Maisse, C.; Daugas, E.; Zamzami, N.; Mak, T.; Jaattela, M.; Penninger, J.M.; Garrido, C., et al. Heat-shock protein 70 antagonizes apoptosis-inducing factor. Nat Cell Biol 2001, 3, 839843, doi:10.1038/ncb0901-839. ncb0901-839 [pii].

19. Samali, A.; Orrenius, S. Heat shock proteins: regulators of stress response and apoptosis. Cell Stress Chaperones 1998, 3, 228-236.

20. Yanaka, A.; Zhang, S.; Sato, D.; Tauchi, M.; Suzuki, H.; Shibahara, T.; Matsui, H.; Nakahara, A.; Hyodo, I. Geranylgeranylacetone protects the human gastric mucosa from diclofenac-induced injury via induction of heat shock protein 70. Digestion 2007, 75, 148-155, doi:000106756 [pii]. 10.1159/000106756.

21. Wang, X.; Yuan, B.; Dong, W.; Yang, B.; Yang, Y.; Lin, X.; Gong, G. Induction of heat-shock protein 70 expression by geranylgeranylacetone shows cytoprotective effects in cardiomyocytes of mice under humid heat stress. PLoS One 2014, 9, e93536, doi:10.1371/journal.pone.0093536. PONE-D-13-47753 [pii].

22. 22. Fan, N.; Yang, G.S.; Lu, J.H.; Yang, N.; Zhang, H.B. Oral administration of geranylgeranylacetone plus local somatothermal stimulation: a simple, effective, safe and operable preconditioning combination for conferring tolerance against ischemia-reperfusion injury in rat livers. World J Gastroenterol 2005, 11, 5725-5731, doi:10.3748/wjg.v11.i36.5725.

23. Tanito, M.; Kwon, Y.W.; Kondo, N.; Bai, J.; Masutani, H.; Nakamura, H.; Fujii, J.; Ohira, A.; Yodoi, J. Cytoprotective effects of geranylgeranylacetone against retinal photooxidative damage. J Neurosci 2005, 25, 2396-2404, doi:25/9/2396 [pii]. 10.1523/JNEUROSCI.4866-04.2005.

24. Hasegawa, M.; Ishiguro, K.; Ando, T.; Goto, H. Geranylgeranylacetone attenuates cisplatin-induced reductions in cell viability by suppressing the elevation of intracellular p53 content without heat shock protein induction. Nagoya J Med Sci 2012, 74, 123-131. 
25. Li, H.; Xu, C.; Li, Q.; Gao, X.; Sugano, E.; Tomita, H.; Yang, L.; Shi, S. Thioredoxin 2 Offers Protection against Mitochondrial Oxidative Stress in $\mathrm{H} 9 \mathrm{c} 2$ Cells and against Myocardial Hypertrophy Induced by Hyperglycemia. Int J Mol Sci 2017, 18, doi:ijms18091958 [pii]. 10.3390/ijms18091958.

26. Sugano, E.; Isago, H.; Murayama, N.; Tamai, M.; Tomita, H. Different anti-oxidant effects of thioredoxin 1 and thioredoxin 2 in retinal epithelial cells. Cell Struct Funct 2013, 38, 81-88, doi:DN/JST.JSTAGE/csf/12025 [pii].

27. Sugano, E.; Murayama, N.; Takahashi, M.; Tabata, K.; Tamai, M.; Tomita, H. Essential role of thioredoxin 2 in mitigating oxidative stress in retinal epithelial cells. J Ophthalmol 2013, 2013, 185825, doi:10.1155/2013/185825.

28. Naoi, M.; Wu, Y.; Shamoto-Nagai, M.; Maruyama, W. Mitochondria in neuroprotection by phytochemicals: bioactive polyphenols modulate mitochondrial apoptosis system, function and structure. Int J Mol Sci 2019, 20, doi:ijms20102451 [pii]. 10.3390/ijms20102451.

29. Tanaka, K.; Iijima, T.; Mishima, T.; Suga, K.; Akagawa, K.; Iwao, Y. Ca(2+) buffering capacity of mitochondria after oxygen-glucose deprivation in hippocampal neurons. Neurochem Res 2009, 34, 221-226, doi:10.1007/s11064-008-9753-2.

30. Boitier, E.; Rea, R.; Duchen, M.R. Mitochondria exert a negative feedback on the propagation of intracellular $\mathrm{Ca}^{2+}$ waves in rat cortical astrocytes. J Cell Biol 1999, 145, 795-808, doi:10.1083/jcb.145.4.795.

31. Gunter, K.K.; Gunter, T.E. Transport of calcium by mitochondria. J Bioenerg Biomembr 1994, 26, 471-485.

32. Patron, M.; Raffaello, A.; Granatiero, V.; Tosatto, A.; Merli, G.; De Stefani, D.; Wright, L.; Pallafacchina, G.; Terrin, A.; Mammucari, C., et al. The mitochondrial calcium uniporter (MCU): molecular identity and physiological roles. J Biol Chem 2013, 288, 10750-10758, doi:R112.420752 [pii]. 10.1074/jbc.R112.420752.

33. Song, J.H.; Shin, M.S.; Hwang, G.S.; Oh, S.T.; Hwang, J.J.; Kang, K.S. Chebulinic acid attenuates glutamateinduced HT22 cell death by inhibiting oxidative stress, calcium influx and MAPKs phosphorylation. Bioorg Med Chem Lett 2018, 28, 249-253, doi:S0960-894X(17)31236-2 [pii]. 10.1016/j.bmcl.2017.12.062.

34. Chuderland, D.; Marmor, G.; Shainskaya, A.; Seger, R. Calcium-mediated interactions regulate the subcellular localization of extracellular signal-regulated kinases. J Biol Chem 2008, 283, 11176-11188, doi:M709030200 [pii]. 10.1074/jbc.M709030200.

35. Li, D.W.; Liu, J.P.; Mao, Y.W.; Xiang, H.; Wang, J.; Ma, W.Y.; Dong, Z.; Pike, H.M.; Brown, R.E.; Reed, J.C. Calcium-activated RAF/MEK/ERK signaling pathway mediates p53-dependent apoptosis and is abrogated by alpha B-crystallin through inhibition of RAS activation. Mol Biol Cell 2005, 16, 4437-4453, doi:E05-01-0010 [pii]. 10.1091/mbc.e05-01-0010.

36. Crompton, M. The mitochondrial permeability transition pore and its role in cell death. Biochem J 1999, 341 ( Pt 2), 233-249. 